\title{
Improving The Performances of WSN Using Data Scheduler and Hierarchical Tree
}

\author{
1R. Jayamma, \\ ${ }^{1}$ Department of Computer Science and Engineering, Madhira Institute of Technology and Science, \\ Kodad, Suryapet dist, Telangana, India. \\ **Corresponding Author: jayarairala@gmail.com
}

Received: 10.02 .2021 , Revised: 05.05.2021, Accepted: 15.07.2021, Published: 16.08 .2021

DOI:

10.53409/mnaa/jcsit/2202

Abstract: Users of data-intensive implementation needs intelligent services and schedulers that will provide models and strategies to optimize their data transfer jobs. Normally sensor nodes are connected to consecutive sensor nodes depending on frequent transmission. To enhance end-to-end data flow parallelism for throughput optimization in high speed WSNs. The major objective is to maximize the WSNs throughput, minimizing the model overhead, avoiding disputation among users and using minimum number of end-system resources. Data packets are broadcasted from sender node to target node. Though, all nodes operate concurrently in various communications, the analysis shows that more packet latencies are occurred and priority-based transmission tasks are performed. Then the proposed Bearing parallelism-based Data Scheduler (BPDS) is used for data scheduling to enhance the end-to-end throughput input parameter. Sensor nodes are fast working node, it verifies each and every node before allocating packet transmission for that node. Busy resources are monitored to inform the nodes that are in processing, based on the schedule it allocates various paths to particular node and monitors the node capacity. Sampling algorithm supports for fixing threshold value, based on the values, they are further allocated to communicate between channels. It assigns the routing path with minimum resources and reduces end to end delay, to improve throughput, and network lifetime.

Keywords: WSN, Bearing Parallelism based Data Scheduler, Busy Resource Allocation, Frequent Data Flowing, Hierarchal Tree Alternative Path.

\section{INTRODUCTION}

ireless sensor networks (WSN) are normally deploying huge amount of sensor nodes, which are capable of recognizing the collected data, and packet broadcasting in overload of wireless routing path. Prearranged network should be utilized as a sector, overload manager, network infrastructure monitor and battleground observation. Wireless Sensor nodes work together in a dispersed, self-governing and self-sorted scheme to end a specific process [1]. It mounds the communication by combining variable wireless networks, which results in uneven characteristics, when over load and fluctuations are occurred. Situation of network becomes critical, since modification in the proposed scheme is required to avoid connection breakdown or intended misbehaviour in the WSNs [2].

Normally wireless sensor network functions below load, it will warn the WSN in reply to an analyzed or identified packet error. Performance report is a creation of more unpredicted packet impulse it transfers minimum amount of data to a target node without lengthily disturbing the sense characteristics of WSN. If huge set of data is been transferred lengthily the data packet is naturally unrestrained and frequently induced in jamming. There are different rules and invented surveys are introduced to reduce traffic rate in WSNs. In an authentic condition, traffic is brought on by intrusion links or packet drop since traffic on the intra channel nodes significantly influence the transmission rates and cause peak energy usage. Traffic rates are accelerated, rebroadcasted, when certain packet in the upper layers or MAC, an occasion that tire out the existing imperfect power of network environment. A minor test operation, exists for managing traffic in WSN [2].

Hence, this kind of congestion directly affects lifetime and has a direct impact in networks. The parameters such as Factor, bit fault, crash and struggle results are obtained in connection with the ranges of traffic rate. Therefore, traffic and transmission rate for sink node is reduced. For this 
method, in order to strengthen transmission rate of target point, crash must be banned by utilize a proper medium access control-based traffic estimation. The traffic occurrence in WSN is made at various levels of connection plan traffic. According to node level traffic, amount of packet received is higher than rate of packet provision, it causes traffic. The traffic happens in most part of sensor nodes that are faster to the target node. Capacity of node traffic increases when packets drop and misuse of energy is carried out in network. Therefore, those kind of traffics honestly losses availability of network and increase network lifetime. While, traffic is control to distinguish with various sectors: first blocking and intrusion identification, and second A rate organize scheme that modifies the rate of performance result. Different parameters are utilized as a part of survey for traffic identification, path failure, packet latency, maximum size of packet. [3].

Traffic is a way taken for managing the transfer inside the network and also to avoid down falls in a network. An incorporated Traffic Control method cannot be normally applied because it incites various main difficulties. Initially, this scheme accelerates overhead of excessive communication in the network that are losses its energy level. In addition, central reports are been used to manage the traffic that performs a poor output reaction to vary in congestion and network conditions [4].

Hierarchal Tree Alternative Path (HTAP) does not consume the network management ability of WSNs performs efficient communication and individual node result creation. Finally, when at communication breakdown, the entire environment should be in functioning. In recent survey, HTAP scheme examines the improvement in exclusive infrastructure, and unbalanced situation. HTAP techniques are activated to seem traffic in wireless sensor network by utilizing alternative ways for the crowded node prevention in the network [5].

The advantage of HTAP scheme is that they are does not add any critical traffic rate to the existing scheme, which is approved for serious traffic in network for traffic occurrence. On the other hand, the problem with HTAP scheme is that the mode packet broadcasting latency to target node among various paths that improves under exact conditions. Study of various transmission channels for all packets is vital to analyze and understand the characteristics and liveliness in a fine-grained networking scheme. In sequence, routes have been used in existing process [5]. HTAP is introduced as a scalable and fruitful technique for performing large scale of broadcasting data packets, and energetic network.

Rest of the paper is constructed as given below. Section II illustrates on related works.
Section III, briefly describe the details about proposed Bearing parallelism-based Data Scheduler (BPDS). It allocates the routing path with frequent data packet movement. Section IV is meant for simulations and performance reports monitored via various parameters. Finally, section V concludes the work with future updates.

\section{RELATED WORK}

Interference-Fault Free Transmission Tree (IFFTT) algorithm was proposed in [6] to achieve a damage free time slot-based communication for each connection in data collection hierarchy, and logically indicate the scheme was best and absolute. Experimental results detected an error occurred in packet transmission. It denotes the fault for packet organized in tree-based hierarchy; whether error arise due to PIs that were not acknowledged by this representation, reproduce into the hierarchical sensor network.

A better path in sensor networks based on the count of hops used for packet forwarding was proposed in [7]. It discovered the optimal routes along with normal Distance Based Linked Kneighborhood Inactive Forecasting (DBLKIF) method. The best possible route has minimum energy usage with maximum data packet transmission rate, optimal path with reduced packet latency. Minimum distance route was obtained by choosing optimal routes along with loop free path. This Novel scheme operated to maintain minimum delay and decreased the energy usage in entire process and rebroadcasting resource usage.

Power Aware Scheduling and Clustering method considering on Ant Colony Optimization (PASC-ASO) was used in [8]. This scheme was meant for less energy consumption by preparing, quantity of nodes in the working state to create packet and maintain node links, when remaining nodes are inactive. Then, ACO scheme was used for forwarding message packet in the network in direct, to reduce the energy exhausted while broadcasting the error data transmitted by sensors in a thickly fixed in environment. This method works as an important position in the improvement of network lifespan by choosing the optimal robust route to achieve the target node.

In [9], an energy-efficient relaying method to conquer the difficulties of WSN was implemented. In this technique it launched a best key by decoupling the route of energy steadiness and data rate altered. Experimental output verified that, in terms of network produced a way, this scheme indistinguishable to the optimal robust output, as compared to greedy algorithms. A packet forecast method in sensor network was used in [10] which could powerfully improve packet delivery ratio through wireless connection. A few schemes have 
chosen for packet allocation of original information to obtain conventional and surrounded point to point delay when meeting the limit of questions, that Nearest Job Next with Combination (NJNC), outperforms in speed assisted data organization with grouping of many requirements serve collectively in a demand manner, without starvation issues as in the case of previous methods were FirstCome-First-Serve (FCFS), Shortest-Job-Next (SJN).

A base station assignment method was proposed in [11] to collect experiences about sensor node thickness in a coverage range at various period depends on these clarifications, it presented candidate base station positions in direct to minimize base station traffic rate. It was continued based on time slot. It was applied in a network simulation distinguish with an additional famous method, specifically GSP-Geographic Sink Placement. It was experimental that the present method exhibited best result with respect to base station traffic rate and also packet drop as distinguished with GSP method. In [12], a Sleep Scheduling and Tree-Based Grouping (SSTBG) method for routing algorithm for energy-efficient in Sensor network was proposed. It conserved energy by stop range like inactive mode of either not possible or redundant nodes that monitor approximately the similar details, depend on the position details to reject unwanted information. Then additionally minimized the energy indulgence of packet transmission in sensor environment, to construct minimum spanning tree with the root as the Cluster Head $(\mathrm{CH})$ from active nodes in a group to broadcast information to sink node.

An Enhanced Dynamic Multilevel Protocol (EDML) for DMP queuing in packet transmission was presented in [13]. This method operated on the notion of transmitting data packets depends on probability. It enhanced the QoS, to prolong network lifespan and monitoring energy usage. Dynamic Multilevel Priority Queue (DMPQ), operated on priority assigned time slot for packet broadcasting. The opportunity of original information was minimized, to keep the characteristics of a network. This condition supported to enhance QoS. An energy efficient communication protocol was implemented in [14] with minimum traffic rate and organization of data packet was also major role for the prolong network lifespan. Present a CRT2FLACO-clustering routing protocol on the basis to control the network traffic with improved the lifetime in WSN. Fuzzy logic supports to obtain reduced the traffic rates compared with previous scheme in sensor infrastructure. This methodology also improved the transmission rate.

A concurrently enhanced fidelity for highintegrity with reduced packet latency for latency receptive was proposed in [15], while the environment was jam-packed. A dynamic queue organization method was performed using fuzzy logic to Quality of Service (QoS) condition in Data Integrity and Delay Differentiated Routing (DIDDR). Experimental output shows that the presented method considerably minimized the packet latency and improved transmission rate distinguish with previous scheme. A scheduler was used in [16], which dedicated a series of successive time period of an access Point to receiving data packet from a sender node, during the time, delay for acknowledgement from the target node in its coverage area, that are communicated to assign a data packet to the neighbor node as an access Point among various routing path. Throughput rate increased based on the selection of each path, which has a highest priority. The Access point may manage communication node, that consumed minimum energy.

\section{PROPOSED METHODOLOGY}

Sensor node senses the information from high speed WSN environment. Here nodes are not updated its position, they are fixed constant. Such high-speed nodes consume more resources. Transmission control protocol provides the efficient communication between source nodes to destination node. Figure 1 shows high Block diagram for bearing parallelism-based data scheduler. High speed sensor nodes operate faster and easy to schedule the communication between source nodes to the target node present in the network. Bearing parallelism-based communication scheme finds the irrelevant data transmission and filter out infrequent data packet transmission. It supports to schedule frequent data broadcasting from one end to another end in network environment. In some cases, while performing a task, time busy resource gives alert signal to relay nodes, it accepts and schedule the transmission task, to enhance the throughput and minimizing packet latency. 


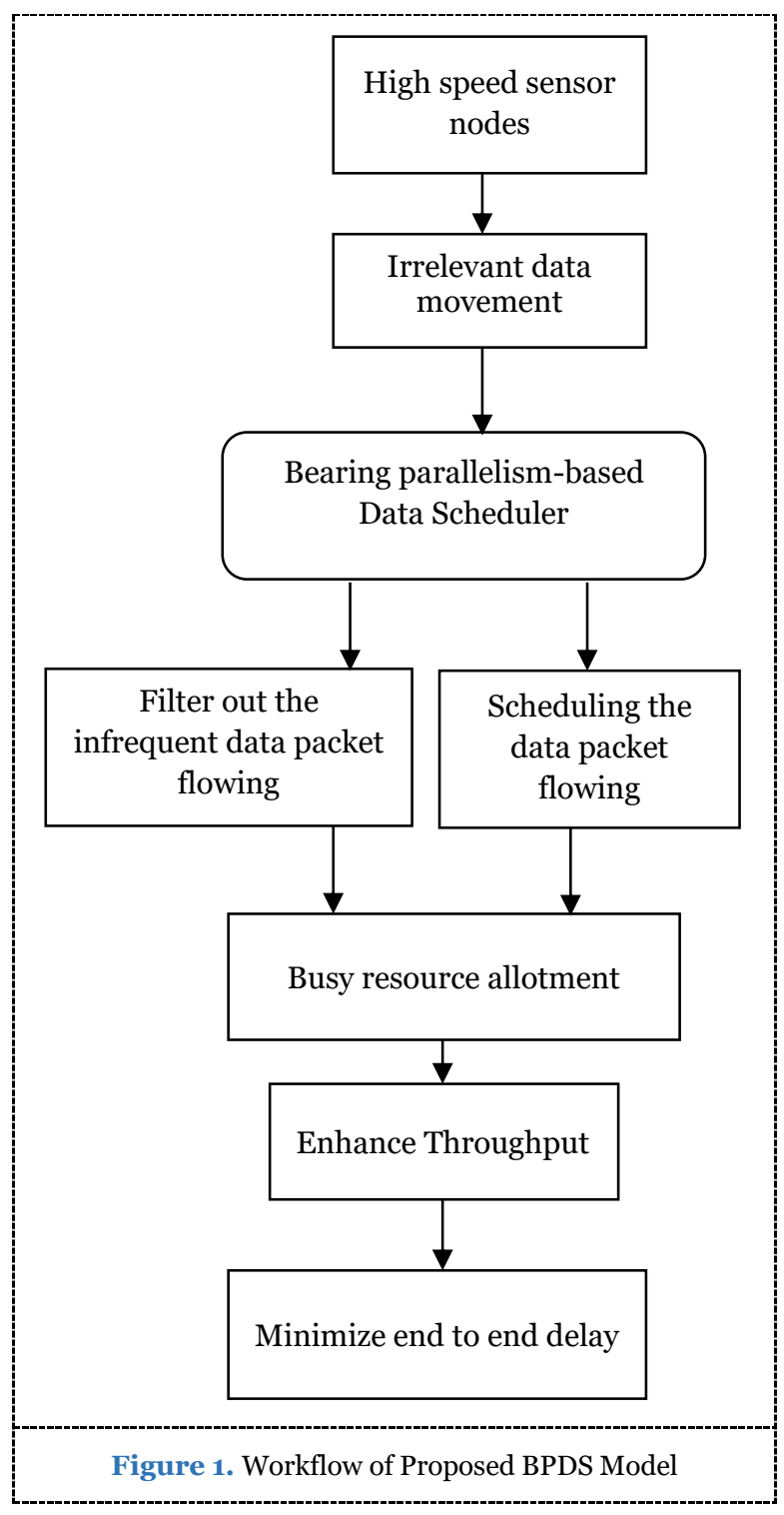

Source sense the normal information from real time environment, and forwards toward the sensed information in destination node. As to find the optimized routing path, there may be huge distortions or losses, which affect the communication, the intermediate node cause delay, since some of nodes may have minimum capacity to transfer frequent data packets to overcome these irrelevant data packet broadcasting to present BPDS.

Normally, nodes bottleneck data packets are forwarded from one end to another end, since it is a high-speed node. If any packet delay that affects the entire communication process, bottleneck data will be lost, the inaccurate data packets are received from receiver node. The missed data are not accurately collected since irrelevant data broadcasting is performed. Present scheme supports to remove infrequent data and obtain frequent data packet transmission.

\subsection{Bottleneck Data Transmission in High- Speed Network}

Sender node sense data, which are bottleneck data packets, they are forwarded through intermediate node to target node. Those intermediate nodes use more resources, so threshold value is fixed, to filter out infrequent data packet transmission based on threshold value. In this technique various defence threshold values are used to preserve intermediate relay nodes. Data packet transmission rate should be equal to each other with guaranteed accurateness, it is a recent scheme to reduce packet latency period.

In this technique, heavy load data packets are transmitted by source node to destination node they use multiple paths, so it consumes more energy for each packet transmission. That information is maintained in the buffer storage place. The buffer conditions are permit status, clean status and discard status, to construct two threshold standards to limit values. Various strategies to accept or decline data packets are adopted in various conditions, by using this scheme. Loss rate is recognized resourcefully with them individually. Data packet probability is a vital role in traffic control, though it does not compensate sufficient concentration. Performance output and the issues are distance-based buffer dissimilarity in the intermediate relay node as the provided resolution is irrelevant data movement for frequent transaction. It works on buffer-based scheduling, transmit data packet as FIFO manner and analyzes the characteristics of subjective equality. The infrequent data transmission causes bandwidth allocation as minimum for nodes at the various penetration of network environment.

$$
\operatorname{FDAT}(n)=\frac{D A T}{D u r}
$$

Where, FDAT is the frequent data and DAT/Dur is the data travel duration. The intermediate relay nodes have more probability to transmit its own created data packets to the destination node. It checks the node capacity before allocating the routing path communication. Though nodes are good in behaviour, it is nearest to the target node which needs to forward its individual data packets among the various hops to knowledge more latency for packet transmission. And provides solution for irrelevant data transmission issues, to introduce comfortable data Scheduler, with the help of queuing system, which distinguish with normally created irrelevant data packet and transmit through infrequent data packet from source node to destination node. All data packets have dual types of probability: constant probability and update probability, that are distinct. Packet constant probability is indicated as an integer and the minimum constant probability node is indicated by 
its packet size zero. Updating probability modifies with the count of hops and packet latency. Whether the traffic made two metrics and are used for edge buffer to improve rate and clean the information.

\section{2. $\quad$ Multipath Bearing Parallelism Based Data Scheduling}

Multipath communication is performed on the explicit or implicit packet messages, which are transmitted from every hop node at the destination energy level of each node. It is maintained as constant range with controlled traffic rate; the source data transmission rate is attuned to mitigate traffic forwarded to destination node from intermediate relay nodes present in network environment. This technique does not provide correct regulated source data transmitting rate. The present scheme states, all nodes can decide communication channel from relay node to target node condition. The $\mathrm{T}_{\mathrm{r}}$ transmitter can discover efficient route to broadcast data packet and the variable source data forwarding rate.

$$
\mathrm{T}_{\mathrm{r}}=\text { INrelay }(\text { Route })
$$

INFDAT-Infrequent data packets are caused based on bottleneck data transmission by high-speed sensor nodes. These nodes operate quickly with every packet transmission, its characteristics are analyzed and stored in buffer. Packets are also maintained in queue buffer, further data packets forwarded in Last in Last out (LILO) manner. Information's are maintained secretly, that cause delay so bearing parallelism-based data scheduler is used to schedule the packet transmission from source node to target node along with the intermediate relay nodes present in network environment.

$$
\operatorname{INFDAT}(n)=D A T / D u r
$$

The individual node capacity is also analyzed it is helpful for further communication between sensor nodes available in WSN. Each node was connected with wireless link, hence coverage range also limited. The threshold value fixed by buffer to separate the node status, some nodes transmit data packet frequent manner that data are accepted, some nodes forward infrequent data packets, such are decline condition. Missing data packet transmission are clear condition of node characteristics, so based on the details to arrange transmission between sensor nodes the throughput rate is improved and end to end delay is reduced.

$$
\begin{aligned}
& \operatorname{INFDAT}(n)=D A T / \mathrm{T}_{\mathrm{r}} \\
& \operatorname{INFDAT}(n)=D A T / I N \text { relay }(\text { Route })
\end{aligned}
$$

All data packets do not broadcast irrelevant data, they are broadcasted based on node capacity, so data scheduler, allocates the routing nodes, the data is allotted without any delay for each packet transmission, time details are taken from a queue buffer, all details are stored in First in First out (FIFO) manner with available link address, based on address, the information of intermediate relay nodes that deployed in network environment. Time period is assigned for each transmission, infrequent data transmission cause packet latency from one end point to another end point. This scheme supports source nodes to broadcast frequent data packet with support of bearing parallelism technique through intermediate relay node to target node.

$$
\operatorname{FDAT}(n)=\frac{D A T}{\text { DINrelay }(\text { Route }) u r}
$$

Source search for best paths

for each source, which monitors various channels

if $\{\mathrm{Sr}==$ Path $\}$

Sr starts to forwards data packet

if $\{$ Node $==$ infrequent packet movement $\}$

Waiting data in queue buffer, $\mathrm{Q}=\mathrm{DAT}$

else

$$
\text { if }\{\text { Node }==\text { frequent packet movement }\}
$$

then it continues packet exchange

the same process will be repeated until it reaches destination node

end if

Source node optimize efficient routing path end if

end for.

\subsection{Busy Resource Allotment}

Sensor network packet transmission is carried out, busy resources are allocated when nodes perform task, and time packet waits for a long period of time for transmission in Queue. The buffer queue stores huge amount of communication and node capacity details in network environment. The path allocation plays a vital role with the support of busy resource allotment scheme. That busy condition gives alert signal to sender node present in network environment. The probability of each sensor intermediate nodes is verified initially and then allocates the packet during particular time slot. It obtains maximum frequent data broadcasting between source nodes to destination nodes.

$$
\begin{aligned}
& D A T(n)=P c K(T x R x) \\
& B s=D A T(n)-\operatorname{INFDAT}(n)
\end{aligned}
$$

$T x R x$ are transmission and receiving packets, maximum probability of neighbour node is selected to perform communication among sensor 
nodes. Minimum probability nodes are rejected and denoted as decline condition. Hence the throughput rate and network lifetime is improved and the energy consumption and end to end delay is minimized.

If $\{$ packet flow $==$ infrequent $\}$ is calculated

Sender node analyses the characteristics of a neighbour node

Allocate busy resources to that node

It creates a busy alert signal

Threshold value is fixed to verify those nodes. Packet transmission is frequent or infrequent

else

If $\{$ packet flow $==$ frequent $\}$

It continues packet transmission on the assigned routing path

Communication is carried on a best routing path

end if

$$
P s=F D A T_{-}(\text {flow })
$$

Sensor nodes sense the information depends on coverage and connectivity rate. All nodes are not easily been connected and communicated with other nodes present in the network environment. It provides the best routing path use busy resource allotment technique. It allocates the busy signal to nearest neighbour node from starting to end point of network communication. It increases the transmission rate.

Packet ID: Packet ID contains each and every sensor node characteristic details. It has nodes that are arranged randomly along the WSN environment.

Table 1. BPDS Packet Format

\begin{tabular}{|c|c|c|c|c|c|}
\hline $\begin{array}{c}\text { Sour } \\
\text { ce } \\
\text { ID }\end{array}$ & $\begin{array}{c}\text { Destina } \\
\text { tion ID }\end{array}$ & $\begin{array}{c}\text { Bottle- } \\
\text { neck } \\
\text { data } \\
\text { transmis } \\
\text { sion }\end{array}$ & $\begin{array}{c}\text { Multip } \\
\text { ath } \\
\text { Bearin } \\
\text { garallel } \\
\text { ism }\end{array}$ & $\begin{array}{c}\text { Scheduli } \\
\text { ng data } \\
\text { packet } \\
\text { transmis } \\
\text { sion }\end{array}$ & $\begin{array}{c}\text { Busy } \\
\text { resour } \\
\text { ce } \\
\text { allocat } \\
\text { ion }\end{array}$ \\
\hline 4 & 4 & 3 & 5 & 6 & 4
\end{tabular}

In table 1, the BPDS packet format is present. The source node ID field occupies 4 bytes and the 4 bytes are occupied by destination node ID field. Third one is Bottleneck data transmission, which is carried out in 3 bytes. When sensor nodes are arranged in fixed environment, node forwards bottleneck data packets. Fourth field Multipath Bearing parallelism consumes 5 bytes and sender works as to select various paths; target node collects the network information through intermediate nodes from sender node. Fifth occupies 6 bytes for scheduling the data packet transmission between sensor nodes present in network environment. The last filed is Enhanced stable channel allotment consumes 3 bytes, to allocate busy resource, routing path among sender to target node in WSN environment.

\section{EXPERIMENTAL RESULTS AND DISCUSSION}

The proposed BPDS scheme is simulated by using Network Simulator tool (NS 2.34). In the simulation, 100 sensor nodes move in a 900-meter $\mathrm{x}$ 800-meter square region for 32 milliseconds simulation time. Each sensor node goes in a random manner along the network in different speed levels. Sensor nodes have coverage area is 250 meters. Constant Bit Rate (CBR) provides a constant speed in packet transmission in networks to limit the traffic rate. Ad-hoc on-Demand Distance vector (AODV) routing protocol is used to obtain enhanced stable channel for packet transmission between senders to sink node. Table 2 indicates Simulation setup.

Table 2. Simulation Setup

\begin{tabular}{|c|c|}
\hline No. of Nodes & 100 \\
\hline Area Size & 900 X 800 \\
\hline Mac & 802.11 \\
\hline Radio Range & $250 \mathrm{Om}$ \\
\hline Simulation Time & $60 \mathrm{~ms}$ \\
\hline Traffic Source & CBR \\
\hline Packet Size & 150 bytes \\
\hline Mobility Model & Random Way Point \\
\hline Protocol & AODV \\
\hline
\end{tabular}

The proposed BPDS scheme, which provides the frequent data broadcasting from source to target node as compared with the existing DQMA, TARA, and TMSP [19-20]. BPDS monitors the node character, node transmits infrequent data packet that cause packet latency. It gives busy alert signal, when time delays occur. Those behaviours are analyzed and further throughput is enhanced and end to end delay is minimized.

Table 3. Pause Time vs End-to-End Delay

\begin{tabular}{ccccc}
\hline \multirow{2}{*}{ Pause Time (ms) } & \multicolumn{4}{c}{ End-to-End Delay (Sec) } \\
\cline { 2 - 5 } & DQMA & TARA & TMSP & BPDS \\
\hline 20 & 23.50 & 15.80 & 13.00 & 11.00 \\
\hline
\end{tabular}




\begin{tabular}{lllll}
\hline 40 & 25.00 & 17.23 & 14.05 & 12.01 \\
\hline 60 & 25.26 & 19.00 & 15.30 & 13.24 \\
\hline 80 & 26.04 & 19.60 & 16.42 & 14.09 \\
\hline 100 & 27.50 & 22.01 & 18.50 & 16.02 \\
\hline
\end{tabular}

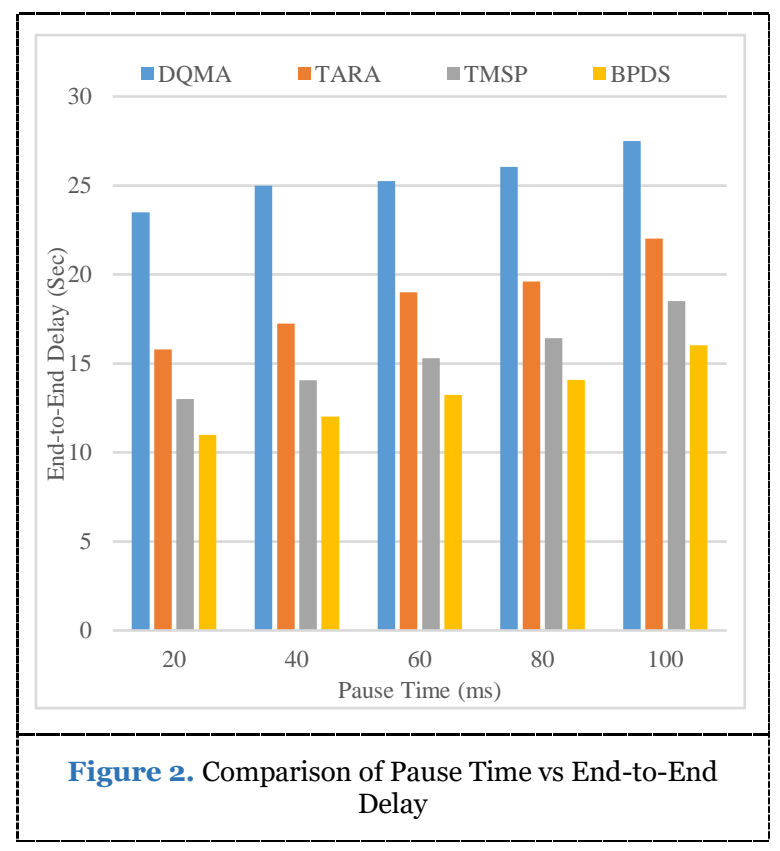

Figure 2 shows the end-to-end delay, calculated based on time taken to forward data packets from source to sink node, all node information is maintained in routing table. In proposed BPDS scheme end to end delay is minimized and distinguished with previous scheme DQMA, TARA, and TMSP.

Table 4. Nodes vs Network Overhead

\begin{tabular}{ccccc}
\hline \multirow{2}{*}{ Nodes } & \multicolumn{4}{c}{ Network Overhead (\%) } \\
\cline { 2 - 5 } & DQMA & TARA & TMSP & BPDS \\
\hline 20 & 53.86 & 41.00 & 28.10 & 23.86 \\
\hline 40 & 54.90 & 43.50 & 29.80 & 24.00 \\
\hline 60 & 55.00 & 45.00 & 31.00 & 26.02 \\
\hline 80 & 57.02 & 46.90 & 32.20 & 29.00 \\
\hline 100 & 59.82 & 47.01 & 33.45 & 30.90 \\
\hline
\end{tabular}

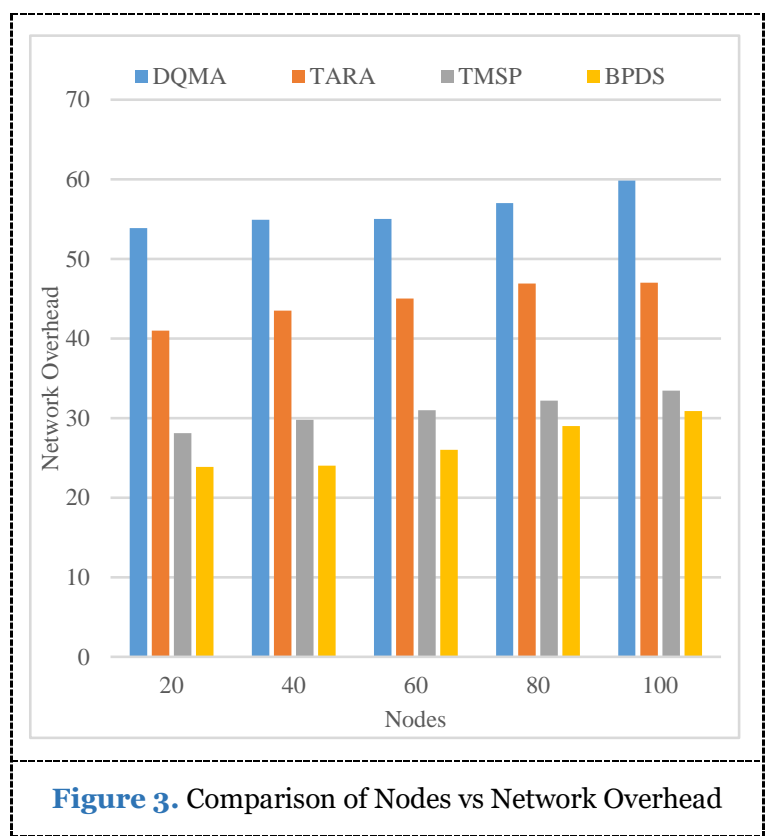

Network overhead is minimized in which the sender transmits the packet to the receiver node. The BPDS controls unwanted packet drops when the sender node initiates its packet transmission along with the network nodes. Fig. 3 and table 4 represents the performance of network overhead. The proposed technique obtained reduced network overhead compared to the existing method DQMA, TARA, and TMSP.

\begin{tabular}{|c|c|c|c|c|}
\hline \multirow{2}{*}{ Nodes } & \multicolumn{4}{|c|}{ Network Overhead (\%) } \\
\hline & DQMA & TARA & TMSP & BPDS \\
\hline 20 & 57.00 & 40.22 & 72.23 & 83.91 \\
\hline 40 & 58.20 & 41.09 & 73.75 & 84.10 \\
\hline 60 & 59.18 & 42.23 & 74.86 & 83.20 \\
\hline 80 & 60.88 & 44.91 & 75.02 & 86.20 \\
\hline 100 & 62.05 & 46.70 & 77.20 & 88.22 \\
\hline
\end{tabular}




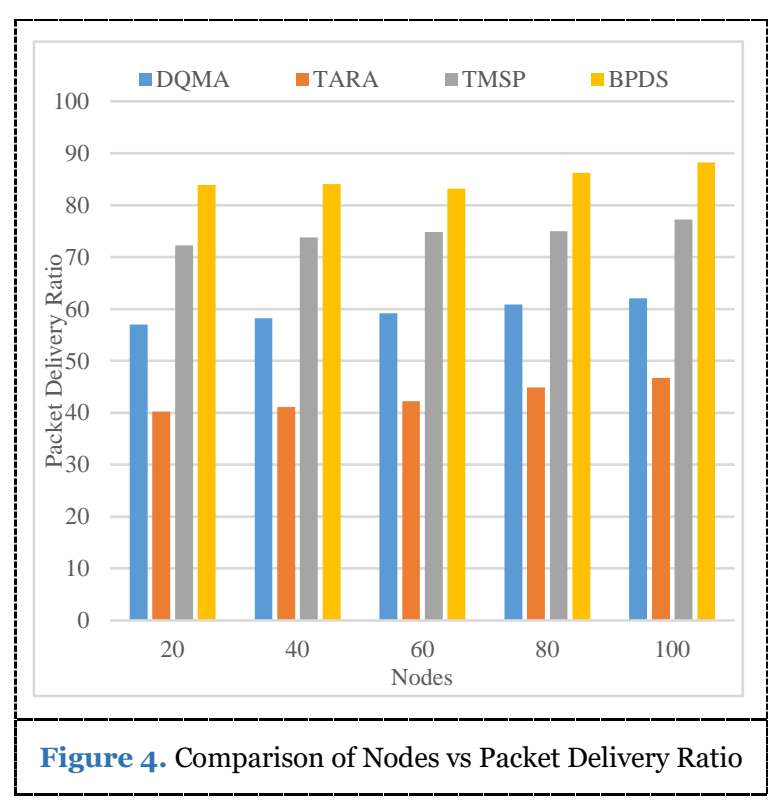

Figure 4 shows Packet Delivery rate, nodes transmit data packet with Overall time taken to finish the particular communication. Those communications take place in an optimal routing path. The proposed BPDS Scheme Packet Delivery rate is increased and compared with existing schemes of DQMA, TARA and TMSP.

Table 6. Mobility vs Network Lifetime

\begin{tabular}{ccccc}
\hline \multirow{2}{*}{ Mobility } & \multicolumn{4}{c}{ Network Lifetime (\%) } \\
\cline { 2 - 5 } & DQMA & TARA & TMSP & BPDS \\
\hline 10 & 31.50 & 48.02 & 68.00 & 83.08 \\
\hline 20 & 33.09 & 50.45 & 69.25 & 83.92 \\
\hline 30 & 35.42 & 52.68 & 70.90 & 84.20 \\
\hline 40 & 36.89 & 53.07 & 72.33 & 84.06 \\
\hline 50 & 38.10 & 55.21 & 74.09 & 85.01 \\
\hline
\end{tabular}

Table 7. Nodes vs Energy Consumption

\begin{tabular}{ccccc}
\hline \multirow{2}{*}{ Nodes } & \multicolumn{4}{c}{ Energy Consumption (J) } \\
\cline { 2 - 5 } & DQMA & TARA & TMSP & BPDS \\
\hline 20 & 411 & 372 & 218 & 128 \\
\hline 40 & 424 & 386 & 225 & 135 \\
\hline 60 & 437 & 401 & 244 & 160 \\
\hline 80 & 448 & 416 & 263 & 174 \\
\hline 100 & 464 & 432 & 270 & 185 \\
\hline
\end{tabular}
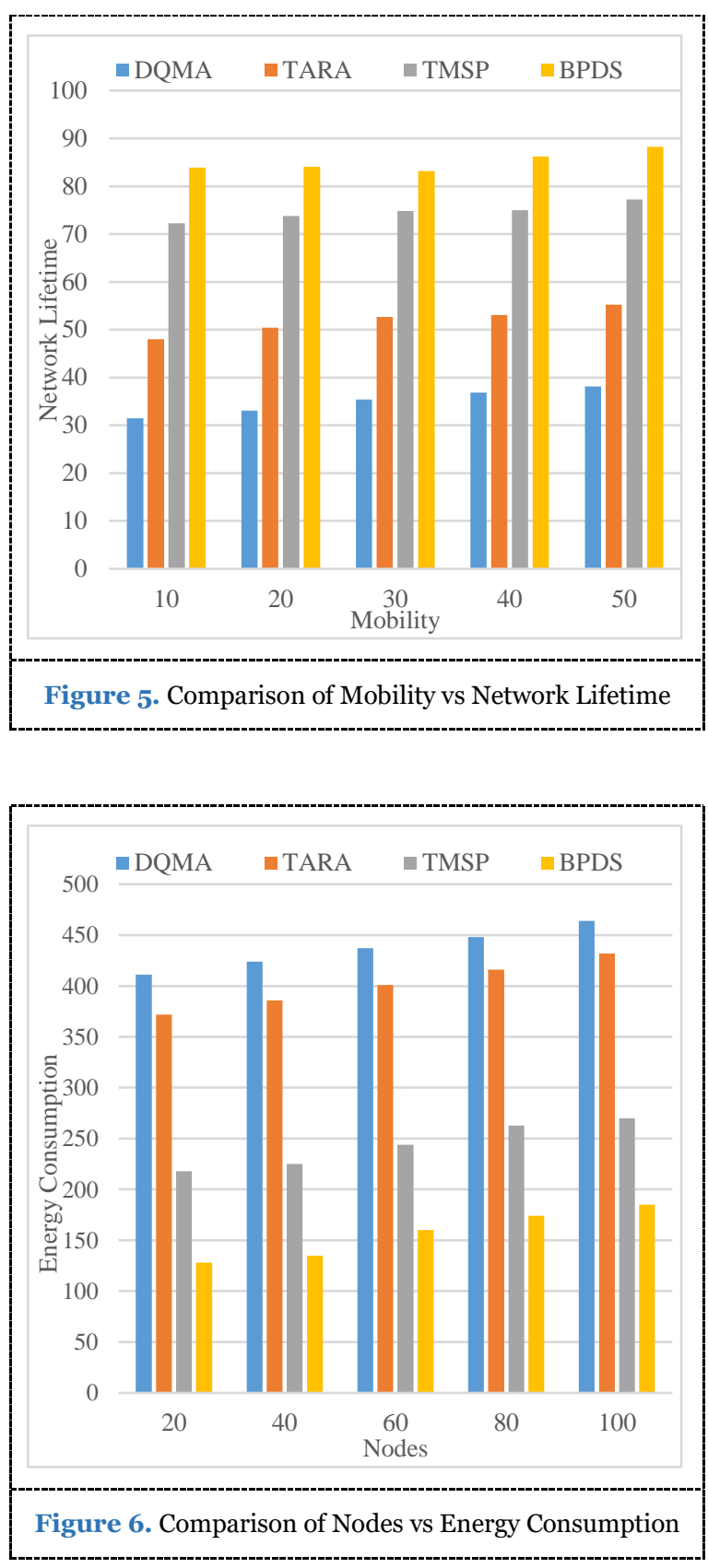

Figure 5 shows the estimated Lifetime of a network, which is computed by calculating the resource utilization at a particular time instance from source to destination from the total energy available. In the proposed BPDS Scheme Network Lifetime is improved as compared to previous schemes of DQMA, TARA and TMSP.

Figure 6 shows the entire energy consumption, amount of energy spends for communication between source nodes to destination nodes that is meant to estimate energy usage from source energy level to destination energy level. The proposed BPDS Scheme have generated an optimal routing path in the network, energy consumption is minimized and distinguished with previous scheme of DQMA, TARA, and TMSP. 
Table 8. Nodes vs Throughput

\begin{tabular}{ccccc}
\hline \multirow{2}{*}{ Nodes } & \multicolumn{4}{c}{ Throughput (\%) } \\
\cline { 2 - 5 } & DQMA & TARA & TMSP & BPDS \\
\hline 10 & 24 & 27 & 59 & 73 \\
\hline 20 & 25 & 29 & 60 & 75 \\
\hline 30 & 27 & 31 & 62 & 76 \\
\hline 40 & 28 & 32 & 64 & 78 \\
\hline 50 & 32 & 35 & 66 & 80 \\
\hline
\end{tabular}

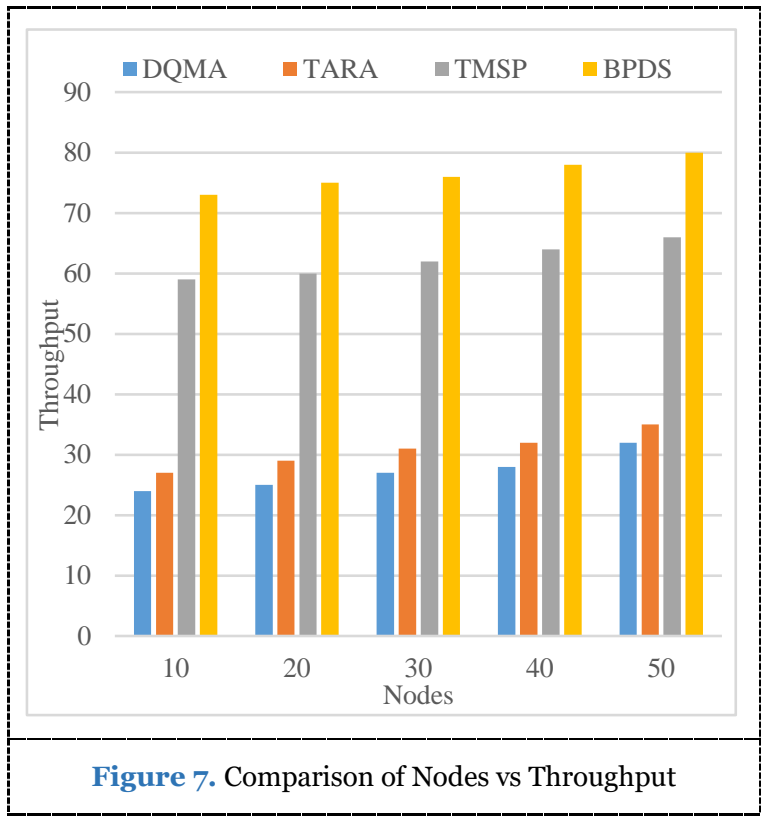

Figure 7 shows the estimated throughput depends on the number of packets received from no of a packet sent with a certain velocity by setting simulation mobility to 100 (bps). In proposing BPDS method throughput is increased as compared with other models like TARA, DQMA, and TMSP.

\section{CONCLUSION AND FUTURE WORK}

Sensor nodes are deployed in a high speed WSN source node, which transmits information to destination node through intermediate nodes, when the data transmission for that node capacity is very low, frequent delays will be occurred for packet transmission. The proposed BPDS scheme provides frequent packet data transmission between source nodes to destination, some abnormal condition nodes provide busy alert due to busy situation. It reduces the end-to-end delay and improve throughput rate. The major objective is to maximize the WSNs throughput, minimizing the model overhead, avoiding disputation among users and using minimum number of end-system resources. Sensor nodes are fast working node, it verifies each and every node before allocating packet transmission for that node. Busy resources are monitored to inform the nodes that are in processing, based on the schedule it allocates various paths to particular node and monitors the node capacity. Sampling algorithm supports for fixing threshold value, based on the values, they are further allocated to communicate between channels. It assigns the routing path with minimum resources and reduces end to end delay, to improve throughput, and network lifetime. In future, this data scheduler is further executed using Fuzzy computing-based communication, to measure the different parameters in WSNs.

\section{ETHICS APPROVAL AND CONSENT TO} PARTICIPATE

Not applicable.

\section{HUMAN AND ANIMAL RIGHTS}

No animals/humans were used for studies that are basis of this research.

\section{CONSENT FOR PUBLICATION}

Not applicable.

AVAILABILITY OF DATA AND MATERIALS The authors confirm that the data supporting the findings of this research are available within the article.

\section{FUNDING}

None.

\section{CONFLICT OF INTEREST}

The authors declare no conflict of interest, financial or otherwise.

\section{ACKNOWLEDGEIMENTS}

The authors would like to thank their present employer for providing support while carrying out this research work.

\section{REFERENCES}

[1]. Al-Aghbari Z, Khedr AM, Osamy W, Arif I, Agrawal DP. Routings in Wireless Sensor Network Using Optimizations Technique: A Survey. Wirel. Pers. Commun., 2020, 111, 24072434.

[2]. Aroba OJ, Naicker N, Adeliyi T. An Innovative Hyperheuristics, Gaussian Clustering Schemes for EnergyEfficient Optimizations in Wireless Sensor Network. J. Sensor., 2021, 2021, 1-12.

[3]. Sridhar R. and Guruprasad N. Energy-efficient chaotic whale optimizations technique for data gatherings in wireless sensor networks. Int. J. Electr. Comput. Eng., 2020, 10(4), 4176-4188.

[4]. Maheshwari P, Sharma AK, Verma K. Energy-efficient clusters-based routings protocols for WSNs using butterfly optimizations algorithm and ant colony optimizations. Ad Hoc Netw., 2021, 110, 102317

[5]. Ajmi N, Helali A, Lorenz P, Mghaieth R. MWCSGA-Multi Weight Chickens Swarm Based Genetic Algorithms for Energy 
Efficient Clustered Wireless Sensors Networks. Sensor., 2021, 21(791), 1-21.

[6]. Begum, Beneyaz A, Satyanarayana VN. Interference-fault free transmission schedule in tree-structured WSN. International Conference on Advances in Computing, Communications and Informatics, 2016, pp. 333-341.

[7]. Kalmegh, M. A, Jain SR. Optimal route discovery in duty cycled wireless sensor network: A review. World Conference on Futuristic Trends in Research and Innovation for Social Welfare, 2016, pp. 1-6.

[8]. Boucetta, Cherifa, Idoudi H, Saidane LA. Ant Colony Optimization based hierarchical data dissemination in WSN. International Wireless Communications and Mobile Computing Conference, 2015, pp. 782-787.

[9]. Li K, Ni W, Wang X, Liu RP, Kanhere SS, Jha S. EPLA: Energy-balancing packets scheduling for airborne relaying networks. IEEE International Conference on Communications, 2015, pp. 6246-6251.

[10]. Gomathi R, Mahendran N. An efficient data packet scheduling schemes in wireless sensor networks. International Conference on Electronics and Communication Systems, 2015, pp. 542-547.

[11]. Banerjee, Subhra, Bhunia SS, Mukherjee N. Experience Based Sink Placement in Mobile Wireless Sensor Network. IEEE/ACM International Symposium on Cluster, Cloud and Grid Computing, 2015, pp. 898-907.

[12]. Tan, Nguyen D, Nguyen D V. SSTBC: Sleep scheduled and tree-based clustering routing protocol for energy-efficient in wireless sensor networks. International Conference on Computing \& Communication Technologies-Research, Innovation, and Vision for the Future, 2015, pp. 180-185.

[13]. Verma S, Paulus R, Jaiswal AK, Nitin N. An enhanced dynamic multilevel protocol (EDMP) for WSN. International Conference on Reliability, Infocom Technologies and Optimization, 2016, pp. 435-440.

[14]. Chavan, Anita, Khiani S. Securely energy aware routing in WSN with efficient clustering. International Conference on Advanced Communication Control and Computing Technologies, 2016, pp. 624-628.

[15]. Kim SW, Jung YG, Shin DR, Youn HY. Dynamic Queue Management Approach for Data Integrity and Delay Differentiated Service in WSN. International Conference on IT Convergence and Security, 2015, pp. 1-5.

[16]. Alayev Y, Chen F, Hou Y, Johnson MP, Bar-Noy A, La Porta $\mathrm{TF}$, Leung KK. Throughput maximization in mobile WSN scheduling with power control and rate selection. IEEE Trans. Wirel. Commun., 2014, 13(7), 4066-4079.

Cite this article as: Jayamma R. Improving the Performances of WSN Using Data Scheduler and Hierarchical Tree. J. Comput. Sci. Intell. Technol. 2021; 2(2): 07-16. (CJCSIT, MNAA PUB WORLD, 2021. 